\title{
PDV-Based Packet Length Allocation for Networked Control Systems
}

\author{
Yun-Bo Zhao, Jongrae Kim, Peng Shi and Guo-Ping Liu
}

\begin{abstract}
Networked control systems typically involve multiple subsystems which share the communication network. In this system setting, besides the optimization of the quality of control of the considered networked control system, the efficient utilization of the communication resources contributes to part of the overall control objective. By realizing the fact that the packet delay variation can be roughly piecewise constant in a relatively large system, a packet length allocation scheme is proposed for this system setting. With the use of this scheme, the performance of the networked control system can be maintained at an acceptable level at the dramatically reduced cost of the communication resources. As a result an optimized balance between the quality of control of the considered networked control system and the quality of service of the communication network can thus be achieved. Simulations illustrate the effectiveness of the proposed scheme.
\end{abstract}

\section{INTRODUCTION}

$\mathbf{N}$ ETWORKED control systems (NCSs) have been attracting more and more attentions from the researchers and engineers in recent decades, mainly due to their distinct features such as easy maintenance, flexible control structure, reduced overall cost, and so on. Although the fast developments of the communication and computing technologies have enabled this redesigned control framework of NCSs, the inclusion of the novel technologies inevitably complicates the design and introduces fundamental issues to both the theories and applications of NCSs. Considerable works have been done and more efforts are still on the way, towards the wide and reliable applications of NCSs in all the related areas in the near future [1]-[3].

Indeed, the so-called communication constraints in NCSs, including, for example, network-induced delay, data packet dropout, data rate constraint, etc. present a challenging difficulty for control engineers and theorists. The main issue is: How can the control performance of NCSs, or the quality of control (QoC), be optimized subject to the communication constraints in NCSs? In this line the communication constraints are typically viewed as given conditions to the control system and therefore conventional control algorithms can be modified without significant difficulties to fit for this

This work was supported by EPSRC research grant EP/G036195/1, and in part by the National Natural Science Foundation of China (NSFC) under Grants 60934006 and 61004020.

Yun-Bo Zhao and Jongrae Kim are with Division of Biomedical Engineering, University of Glasgow, Glasgow, G12 8QQ, UK (e-mail: \{YunBo.Zhao, Jongrae.Kim\} @glasgow.ac.uk)

Peng Shi and Guo-Ping Liu are with Faculty of Advanced Technology, University of Glamorgan, Pontypridd, CF37 1DL, UK (e-mail: \{pshi,gpliu\}@glam.ac.uk). Peng Shi is also with School of Engineering and Science, Victoria University, Melbourne, Vic 8001, Australia. new problem configuration. Numerous works have been done from various perspectives in control theory, including but not limited to, time delay system theory [4], [5], switch system theory [6], [7], sampled-data system theory [8], [9], etc. Some other works consider also the controller design with the optimal utilization of the given communication resources, which enables a control strategy named "co-design" for NCSs [10].

A shared foundation of these existing works is that the considered NCS has been actually isolated from the whole system: The communication conditions are given a prior and thus NCSs can be modeled and analyzed from merely the control system perspective. Although this "isolation" strategy significantly simplifies the problem setting, a global view of NCSs however provides us with a very different perspective of NCSs. Indeed, the design of a specific NCS should never be too selfish: Despite the desire of optimizing the control objective of this NCS, the NCS should also be responsible for the overall quality of service (QoS) of the communication network it uses. Bearing this in mind, we realize that an optimal design of NCSs ought to consume as less of the communication resources as possible besides the optimization of the QoC. This optimization will then ensure that the performances of other related systems can also be optimized or at least not severely deteriorated. This global view thus proposes a novel problem of designing NCSs subject to the balance between the QoC of the NCS and the QoS of the communication network.

In this work we consider an NCS configuration where the data packets used to send the control signals are shared with other applications. For such a system setting, the communication resources can be measured by the data size required to encode the control signal and two extreme cases are observed in terms of the usage of the communication resources. Most conventional control algorithms use only limited data size that are able to encode one single step of the control signal while a recently reported packet-based control approach uses a much larger data size that is able to encode multiple steps (the upper bound of the round trip delay) of the control signals [10]-[12]. A further observation of the delay in NCSs reveals a fact that the packet delay variation (PDV) has roughly a piecewise constant feature. Taking advantage of this feature we design a novel PDV-based packet length allocation (PDV-PLA) scheme, using which we are able to show that the usage of the communication resources can be dramatically reduced while at the same time the system performance can be maintained at an acceptable level. Unlike either conventional control algorithms or the packet- 
based control approach, the PDV-PLA scheme allocates the data size required by the NCS according to the network conditions. It is also understood that under the PDV-PLA scheme both conventional control algorithms and the packetbased control approach can be appropriately fitted as its two extreme cases, thus making the PDV-PLA scheme a unified framework in terms of the usage of the communication resources.

The remainder of the paper is organized as follows. The problem of interest is formulated in Section II. The PDVbased packet length allocation scheme is then proposed in Section III, on the basis of an important observation of the communication characteristics in the considered system setting. A model predictive controller is designed in Section IV for the completeness of the proposed scheme. Both the PDV-PLA scheme and the controller design method are validated in Section $\mathrm{V}$ using numerical examples and Section VI concludes the paper.

\section{Problem FORMulation}

The system considered in the present work is illustrated in Fig. 1, which is a large scale system consisting of multiple subsystems and the data exchanges of the subsystems are through the same communication network. Within this large scale system, what we are particularly interested in is the NCS shown on the right hand of the figure, which shares the communication resources with other subsystems. Despite our focus on the NCS, the design and implementation of the NCS have to be put in the context of the large, interconnected system of subsystems. This global perspective thus makes the problem setting different from most existing endeavors in NCSs.

The following linear nominal system model for the plant is adopted,

$$
x(k+1)=A x(k)+B u(k)
$$

where $x \in \mathbb{R}^{n}, u \in \mathbb{R}^{m}, A \in \mathbb{R}^{n \times n}, B \in \mathbb{R}^{n \times m}$, and the state is assumed to be available to the controller directly and thus the measurement system is not necessary. It is worth pointing out that the use of the simple plant model in (1) is merely due to the considerations of simplifying the analysis and concentrating mainly on the design of the PDVPLA scheme. Indeed, the design of the PDV-PLA scheme is independent from the plant models, meaning that all the plant models can be fitted within this framework and the use of the plant model in (1) is without loss of generality.

In what follows, we first discuss the balance between the control and communication requirements in such a system setting and then point out two extreme cases in terms of the usage of the communication resources in NCSs. These discussions motivate the design of the PDV-PLA scheme in the following section.

\section{A. Balance between the control and communication require- ments}

It is seen from Fig. 1 that the communication network is not private to the considered NCS but shared with other

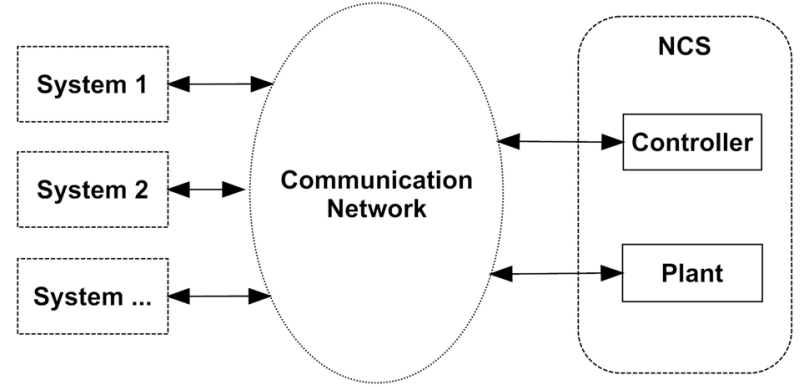

Fig. 1. Networked control systems which share the limited communication resources with other applications.

applications. In addition, these applications can be various and may not be solely for the control purpose. In order to quantitatively measure how the communication network is shared, we consider the scenario where the payload of the data packets in the communication network are filled with both the control data of the NCS sent from the controller to the actuator and the data required by other applications, as illustrated in Fig. 2. That is, whenever a control signal is produced, it is packed into a data packet with the rest of the payload being filled with the data from other applications. The data packet is then sent to the actuator where the control data is extracted from it to close the NCS.

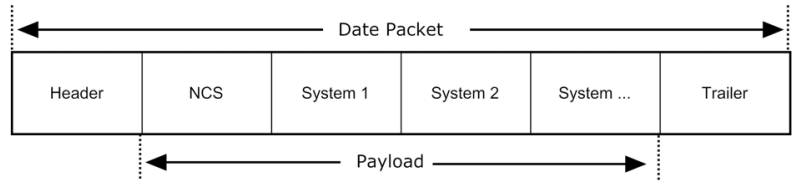

Fig. 2. The payload of the data packet is shared between the networked control system and other applications.

On the one hand, from the perspective of control engineering a controller is designed for the NCS to meet certain control objectives, or in other words, the QoC. The control objectives can be the stability, robustness, or some other optimization indices of the NCS. Numerous conventional control algorithms have already been applied to achieve this goal. It is noticed that in these works the characteristics of the communication network are typically given a prior as certain parameters to the control system, and thus the optimization of the communication network is not part of this control objective.

On the other hand, however, if we look at the system setting in Fig. 1 and Fig. 2 from a global point of view, it is realized that an optimized controller design for the NCS should not only optimize the required QoC of the NCS, but also try to use as little of the payload of the data packet as possible in order that the performances of other applications can also be optimized or at least not degraded too much. In this sense the design and implementation of the NCS have to be considered at a higher level with a two-fold objectives involving not only the QoC of the NCS but the QoS of the communication network. This demand thus makes the 
current problem setting different from most existing ones.

We write the overall objective of the considered problem in an clearer way as

$$
\max Q o C \text { and } \min Q o S
$$

and state the problem of interest as follows.

Problem: Design appropriate control strategy for the NCS shown in Fig. 1 with the plant model in (1) such that the combined objective in (2) can be optimized.

\section{B. Usage of the payload: Two extreme cases}

In terms of the usage of the payload of the data packets as illustrated in Fig. 2 by existing control algorithms, two extreme cases are observed. In most conventional control strategies, the controller typically produces and sends a single step of the control signal at each step, which is clearly the minimum possible usage by the NCS,

$$
B_{\min }=: B_{c}
$$

where $B_{c}$ represents the data size required for encoding a single step of the control signal for the considered NCS, and $B_{\text {min }}$ represents this minimum usage.

A recently reported packet-based control approach improves the system performance by using a larger amount of the payload. Specifically, using this approach at every time step $(\bar{\tau}+1)$ control predictions are packed into a data packet and sent simultaneously to the actuator, i.e.,

$$
B_{\max }=:(\bar{\tau}+1) B_{c} \leq B_{p}
$$

where $\bar{\tau}$ is the upper bound of the round trip delay, $B_{p}$ is the payload of the data packet and $B_{\max }$ represents this maximum usage. Here we have made a reasonable assumption that encoding any single step of the control signal consumes the same amount of the data size.

The enormous advantage of using $B_{\max }$ in the packetbased control approach rather than $B_{\min }$ in conventional control algorithms is that the communication constraints in NCSs can now be actively compensated for, which can never be achieved using the latter. This achievement is made at the cost of dramatically increased usage of the communication resources. Although this increased usage can be no harm in the presence of abundant communication resources, e.g., the communication network is private to the NCS; In the present system setting the demand of optimizing the overall QoS does present a challenge and novel approaches and certain trade offs are therefore necessary. This justifies the PDV-PLA scheme proposed in the present work, which will be detained in the following section.

\section{NCSS WITH THE PDV-PLA SCHEME}

The PDV-PLA scheme is presented in this section. The implementation of the scheme is based on an observation on the communication characteristics in the considered system setting, which is firstly discussed in detail, as follows.

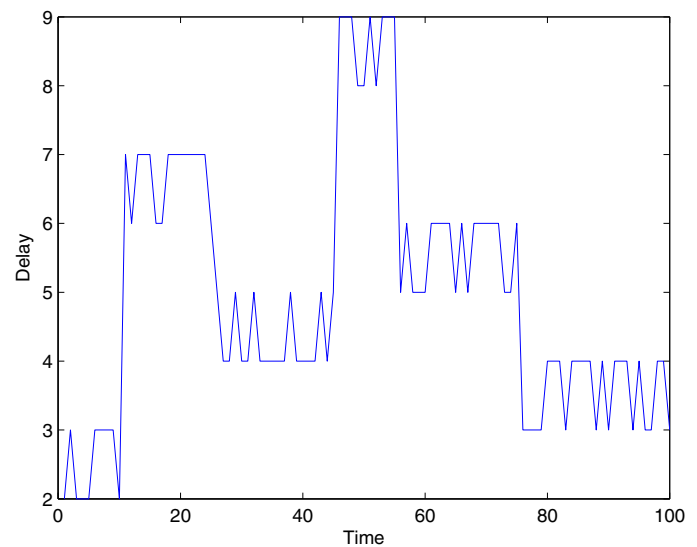

Fig. 3. Illustrating the piecewise constant feature of the delay.

\section{A. Observations on the communication characteristics}

Before proceeding with the observation on the communication characteristics as mentioned before, we first present the following assumption which is also essential in the implementation of the PDV-PLA scheme.

Assumption 1: The data exchanges in the sensor-tocontroller channel and the controller-to-actuator channel are endowed with the similar communication characteristics.

The underlying motivation of the above assumption is that the data packets from the sensor to the controller and from the controller to the actuator are transmitted through the same communication network, as shown in Fig. 1. With this network configuration, Assumption 1 is thus equivalent to say that at any specific time a data packet transmitted from some node $N_{A}$ to some other node $N_{B}$ will experience the same (similar) possibility of delay, dropout, etc. as that transmitted from $N_{B}$ to $N_{A}$, which obviously makes sense.

Consider now the PDV of the communication network in Fig. 1, where "PDV", or packet delay variation, defined in ITU-T Recommendation Y.1540, is the difference in end-toend delay in a data flow with any lost packets being ignored [13]. It is realized that in the current system setting there are two main sources that contribute to a large PDV: The joining and leaving of subsystems and the bursting of the data exchanges of certain subsystems. Without the two sources, the PDV can still be variant but will not change significantly with a high probability. Note the fact that in practice the appearance of the two aforementioned situations will both last for a fairly long time, it is thus reasonable to see a typical PDV as approximately piecewise constant. That is, the delay remains approximately constant for a certain time period, changes suddenly whenever one of the two aforementioned main sources takes effect, and then remains approximately constant at another lever again, as illustrated in Fig. 3. This observation is an important basis of implementing the PDV-PLA scheme, and is stated formally in the following assumption. 
Assumption 2: The PDV of the communication network in the system as illustrated in Fig. 1 is approximately piecewise constant.

\section{B. NCSs with the PDV-PLA scheme}

The observations made in the last subsection actually provide us with the possibility of estimating the delay in the controller-to-actuator channel based on the delay information in the sensor-to-controller channel, which is the fundamental basis of the proposed PDV-PLA scheme. Indeed, upon knowing the delay in the sensor-to-controller channel, Assumption 2 ensures that this delay will remain unchanged, or more precisely, change but within a small range of variation with a high probability for a relatively long time period. Then, Assumption 1 implies that this particular delay level will also apply to the controller-to-actuator channel. Hence, we now have a reasonable estimation of the delay in the controllerto-actuator channel and the PDV-PLA scheme can then be designed by taking advantage of it, as follows.

Firstly, the sampled data packets sent from the sensor to the controller are time stamped, a technique widely used in NCSs [11]. With the use of time stamps, the delay in the sensor-to-controller channel can then be measured, provided that the controller and the sensor are time synchronized. Thus we are able to obtain a reasonable estimation of the delay in the controller-to-actuator channel, as follows, if no switch of the delay levels occur during the following time interval,

$$
\hat{\tau}_{c a, k} \in\left[\tau_{s c, k}-\tau_{\delta}, \tau_{s c, k}+\tau_{\delta}\right]
$$

where $\tau_{\delta}$ represents the variation of the PDV, $\tau_{s c, k}$ is the measured delay in the sensor-to-controller channel and $\hat{\tau}_{c a, k}$ is the estimation of the delay in the controller-to-actuator channel, both are measured or estimated at time $k$ at the controller side.

Then, with $\hat{\tau}_{c a, k}$ the controller is able to produce a forward control sequence (FCS) at time $k$, which consists of $2 \tau_{\delta}+$ 1 control predictions starting from time $k+\tau_{s c, k}-\tau_{\delta}$, as follows,

$$
\begin{aligned}
& U\left(k \mid k-\tau_{s c, k}\right)=\left[u\left(k+\tau_{s c, k}-\tau_{\delta} \mid k-\tau_{s c, k}\right)\right. \\
& \left.\quad \ldots u\left(k+\tau_{s c, k} \mid k-\tau_{s c, k}\right) \ldots u\left(k+\tau_{s c, k}+\tau_{\delta} \mid k-\tau_{s c, k}\right)\right]
\end{aligned}
$$

where all the control predictions are calculated based on the state information $x\left(k-\tau_{s c, k}\right)$. In the present work we consider the use of state feedback, and thus the general form of the FCS in (6) can be modified as follows,

$$
\begin{aligned}
& U_{x}\left(k \mid k-\tau_{s c, k}\right)=\left[x\left(k-\tau_{s c, k}\right)\right. \\
& K\left(k+\tau_{s c, k}-\tau_{\delta} \mid k-\tau_{s c, k}\right) \ldots K\left(k+\tau_{s c, k} \mid k-\tau_{s c, k}\right) \\
& \left.\ldots K\left(k+\tau_{s c, k}+\tau_{\delta} \mid k-\tau_{s c, k}\right)\right]
\end{aligned}
$$

where $K\left(k+\tau_{s c, k}+i \mid k-\tau_{s c, k}\right), i=-\tau_{\delta}, \ldots, \tau_{\delta}$ are the feedback gains with respect to different delays in the controller-to-actuator channel. The control signals as in (6) can be constructed for $i=-\tau_{\delta}, \ldots, \tau_{\delta}$ as follows,

$$
\begin{aligned}
& u\left(k+\tau_{s c, k}+i \mid k-\tau_{s c, k}\right) \\
= & K\left(k+\tau_{s c, k}+i \mid k-\tau_{s c, k}\right) x\left(k-\tau_{s c, k}\right)
\end{aligned}
$$

Finally, a different strategy compared with the packetbased control approach is adopted at the actuator side in order to implement the PDV-PLA scheme successfully. In packetbased control approach, a control action selector is designed at the actuator side to select the appropriate control signal from the FCS according to current network condition. This strategy can be implemented since the FCS used in the packetbased control approach ensures that the appropriate control signal is always available from the FCS to compensate for the communication constraints, which however is not the case using the FCS in (6). Indeed, if whenever the difference of the real delay in the controller-to-actuator channel, $\tau_{c a, k}$, and the estimated delay, $\hat{\tau}_{c a, k}$, is larger than $\tau_{\delta}$, then no control prediction can be found in the current FCS. In order to deal with this issue, we design a static feedback gain $K$ offline without the consideration of different network conditions, as typically done in conventional algorithms. This is used as a complement of the PDV-PLA scheme. Specifically, a "gain scheduler" is implemented at the actuator side which is in charge of the appropriate selection of the control signal. At time $k$ at the actuator side (when the available FCS was produced at time $k-\tau_{c a, k}$ at the controller side), the gain scheduler determines the control action by the following rule,

$$
u(k)= \begin{cases}K\left(k \mid k-\tau_{k}\right) x\left(k-\tau_{k}\right), & \left|\tau_{c a, k}-\tau_{s c, k}\right| \leq \tau_{\delta} \\ K x\left(k-\tau_{k}\right), & \text { otherwise }\end{cases}
$$

where $\tau_{k}=: \tau_{s c, k}+\tau_{c a, k}$ is the round trip delay. That is, the designed static feedback gain closes the system whenever the PDV-PLA scheme fails to do so.

The PDV-PLA scheme for NCSs can now be organized as follows, with its schematic structure being depicted in Fig. 4.

Algorithm 1 (NCSs with the PDV-PLA schme):

$\mathrm{S} 1$. The sensor samples the plant and sends the sampled data to the controller;

$\mathrm{S} 2$. The delay estimator estimates the delay in the controller-to-actuator channel by (5), based on which the controller produces the FCS by (7), and sends it to the actuator;

S3. The actuator applies the control signal determined by (9) to the plant.

Remark 1 (Dropout and disorder): Although not particularly pointed out, data packet dropout and disorder can also be treated within the PDV-PLA scheme, similarly as done in classic packet-based control approach. These communication constraints typically increase the variant of the PDV, $\tau_{\delta}$, and consequently increase the use of the static feedback gain $K$ rather than the delay-dependent one $K\left(k \mid k-\tau_{k}\right)$ in (9). The reader is referred to [11], [14] for more details of the treatment of data packet dropout and disorder within the packet-based control framework.

Remark 2 (Flexibility of Algorithm 1): The only prerequirement of the implementation of the PDV-PLA scheme is the estimation of the delay in the controller-to-actuator channel. In the current system setting the delay is estimated based on Assumptions 1 and 2 in Subsection III-A. It is 


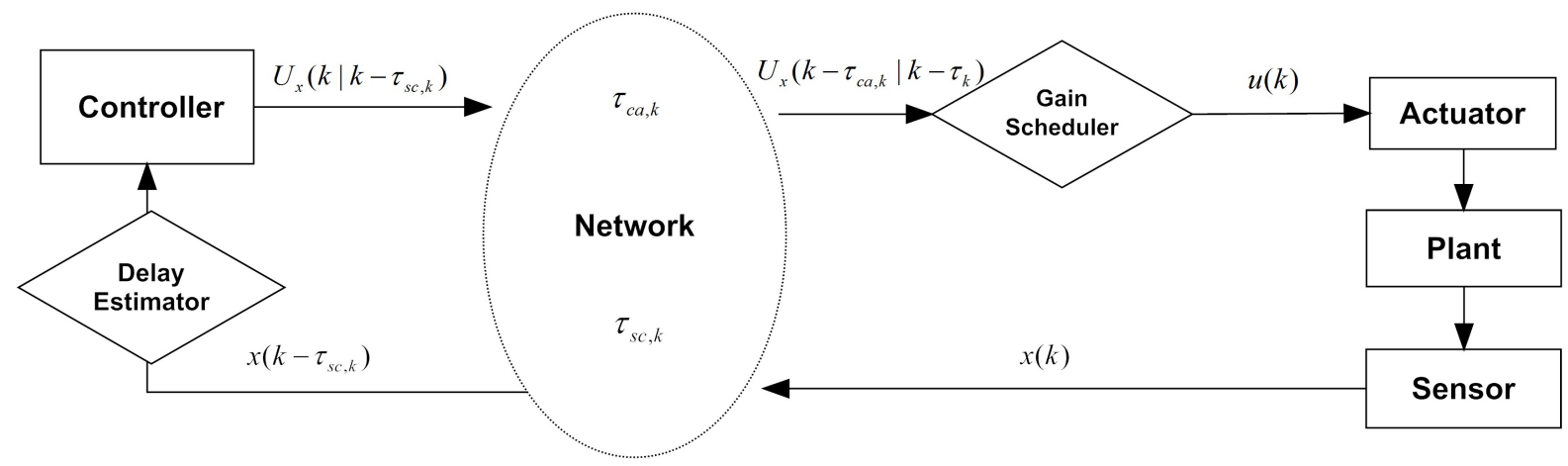

Fig. 4. Networked control systems with the dynamic packet length allocation scheme.

worth pointing out that the PDV-PLA scheme can still be applied without significant modifications when any other estimation methods are available, which thus makes the implementation of Algorithm 1 fairly flexible.

Remark 3 (Usage of the payload): The following relationship regarding the usage of the payload can be established,

$$
B_{\min }: B_{m}: B_{\max }=1:\left(2 \tau_{\delta}+1\right):(\bar{\tau}+1)
$$

where $B_{m}$ represents the usage of the payload with the PDV-PLA scheme. Notice the fact that $\tau_{\delta}$ can be much smaller than $\bar{\tau}$, it is thus not difficult to conclude that the PDV-PLA scheme can significantly reduce the usage of the payload compared with the packet-based control approach. In addition, conventional control algorithms and the packetbased control approach can be regarded as two extreme cases in terms of the usage of the payload, if we simply set $\tau_{\delta}=0$ and $\tau_{\delta}=\bar{\tau} / 2$, respectively. This thus implies that the PDVPLA scheme can be regarded as a unified framework in terms of the usage of the communication resources in NCSs.

\section{Model PREDictive CONTROLLER DESIGN}

The PDV-PLA scheme proposed in the last section is a general control framework for the system setting in Fig. 1 , in the sense that it admits all the appropriate controller design methods to produce the FCS, provided these methods can give rise to a desirable system performance. We design an model predictive control (MPC) based controller in this section for an example.

MPC is a finite horizon optimal control strategy. It solves a finite horizon oppilation problem at every step, and then the first control input of the resulting control predictions is applied to the control system while others are discarded. The optimization problem is solved at every time step, enabling MPC to deal with noises, uncertainties and constraints at an affordable cost.

In our problem setting the objective function of MPC at time $k$ at the controller side, denoted by $J_{k}$, is designed as follows,

$$
\begin{aligned}
J_{k}= & X^{T}\left(k \mid k-\tau_{s c, k}\right) Q_{\tau_{s c, k}} X\left(k \mid k-\tau_{s c, k}\right) \\
& +U^{T}\left(k \mid k-\tau_{s c, k}\right) R_{\tau_{s c, k}} U\left(\mid k-\tau_{s c, k}\right)
\end{aligned}
$$

where $U\left(k \mid k-\tau_{s c, k}\right)=\left[u\left(k-\tau_{s c, k} \mid k-\tau_{s c, k}\right) \ldots u(k+\right.$ $\left.\left.N-1 \mid k-\tau_{s c, k}\right)\right]^{T}$ is a sequence of the control predictions to be determined, $X\left(k \mid k-\tau_{s c, k}\right)=\left[x\left(k-\tau_{s c, k}+1 \mid k-\right.\right.$ $\left.\left.\tau_{s c, k}\right) \ldots x\left(k+N \mid k-\tau_{s c, k}\right)\right]^{T}$ is the predictive state trajectory, $Q_{\tau_{s c, k}}$ and $R_{\tau_{s c, k}}$ are weighting matrixes with appropriate dimensions and $N$ is the prediction horizon. In the current system setting, it is required that $N>\tau_{s c, k}+\tau_{\delta}$.

In order to solve the optimization problem subject to the system in (1) and the objective in (11), the predictive states at time $k$ can be firstly obtained by iteration for $j=1,2, \cdots, N+\tau_{s c, k}$, as follows

$$
\begin{gathered}
x\left(k-\tau_{s c, k}+j \mid k-\tau_{s c, k}\right)=A^{j} x\left(k-\tau_{s c, k}\right) \\
+\sum_{l=0}^{j-1} A^{j-l-1} B u\left(k-\tau_{s c, k}+l \mid k-\tau_{s c, k}\right)
\end{gathered}
$$

where $u\left(k-\tau_{s c, k} \mid k-\tau_{s c, k}\right)=u\left(k-\tau_{s c, k}\right)$, and both $u(k-$ $\left.\tau_{s c, k}\right)$ and $x\left(k-\tau_{s c, k}\right)$ are available to the controller.

Hence the predictive states in the vector form can be constructed as

$X\left(k \mid k-\tau_{s c, k}\right)=E_{\tau_{s c, k}} x\left(k-\tau_{s c, k}\right)+F_{\tau_{s c, k}} U\left(k \mid k-\tau_{s c, k}\right)$

where $E_{\tau_{s c, k}}=\left[A^{T} \cdots\left(A^{N+\tau_{s c, k}}\right)^{T}\right]^{T}$ and $F_{\tau_{s c, k}}$ is a $(N+$ $\left.\tau_{s c, k}\right) \times\left(N+\tau_{s c, k}\right)$ block lower triangular matrix with its non-null elements defined by $\left(F_{\tau_{s c, k}}\right)_{i j}=A^{i-j} B, j \leq i$.

The optimal FCS can then be calculated by substituting (12) to (11) and minimizing $J_{k}$, which turns out to be state feedback control, as follows,

$$
U\left(k \mid k-\tau_{s c, k}\right)=K_{\tau_{s c, k}} x\left(k-\tau_{s c, k}\right)
$$

with the feedback gain vector $K_{\tau_{s c, k}}$ being defined by

$K_{\tau_{s c, k}}=-\left(F_{\tau_{s c, k}}^{T} Q_{\tau_{s c, k}} F_{\tau_{s c, k}}+R_{\tau_{s c, k}}\right)^{-1} F_{\tau_{s c, k}}^{T} Q_{\tau_{s c, k}} E_{\tau_{s c, k}}$

Finally, the FCS as in (7) can be constructed by selecting appropriate items from the feedback gain vector $K_{\tau_{s c, k}}$ defined in (14).

Remark 4: The requirement of $N>\tau_{s c, k}+\tau_{\delta}$ is to ensure that the gain predictions from $k+\tau_{s c, k}-\tau_{\delta}$ to $k+\tau_{s c, k}+$ $\tau_{\delta}$ are available from (14). A larger $N$ results in a larger prediction horizon and thus usually provides a better system 


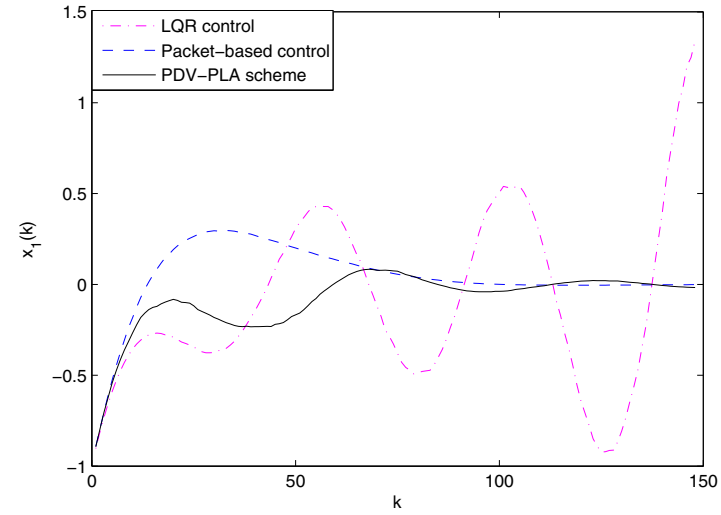

Fig. 5. State evolutions of the networked control system, showing only $x_{1}$.

performance. However the computational complexity of the MPC algorithm is highly dependent on $N$ and thus a properly selected $N$ is subject to the constraints of both the system performance and the computation resources.

\section{NUMERICAL EXAMPLES}

Consider the system in (1) with the following system matrices borrowed from [11],

$$
A=\left(\begin{array}{cc}
0.98 & 0.1 \\
0 & 1
\end{array}\right), B=\left(\begin{array}{c}
0.04 \\
0.1
\end{array}\right) .
$$

which is open-loop unstable.

The initial state for the above system is set as $x_{0}:=$ $\left[x_{1}(0) x_{2}(0)\right]^{T}=\left[\begin{array}{ll}-1 & 1\end{array}\right]^{T}$. The upper bounds of the delay in the sensor-to-controller and the controller-to-actuator channels are both set as 8 time steps, with the variation of the PDV being $\tau_{\delta}=2$. In order to demonstrate the effectiveness of the PDV-PLA scheme, we consider the comparison of the PDV-PLA scheme with 1) A linear quadratic regulator (LQR) for the system with the controller being given by $K=[-0.6893-1.2618]$, and 2) a packet-based controller obtained as in Section IV, using which a FCS with 17 (where $\bar{\tau}=16)$ control predictions are produced and sent to the actuator at every time step.

The state evolutions of the system under the LQR controller, the packet-based control approach and the PDVPLA scheme are illustrated in Fig. 5, where the PDV-PLA scheme is implemented with the use of both the MPC-based controller discussed in Section IV and the aforementioned LQR controller. It is seen that the state evolution with the LQR controller is unstable, but the evolutions are stable with the packet-based control approach and the PDV-PLA scheme. Furthermore, the system response with the PDVPLA scheme is fairly acceptable despite its a bit more fluctuation compared with the packet-based control approach.

At the meanwhile, with the use of the PDV-PLA scheme, the FCS used is constructed by $2 \tau_{\delta}+1=5$ control predictions at every time step. Compared with the packetbased control approach where 17 control predictions are included in the FCSs, the PDV-PLA scheme thus reduces more than $70 \%$ of the communication resources consumed by the packet-based control approach. We are therefore confident to conclude that the PDV-PLA scheme can be an efficient approach towards the optimal balance of the combined objective in (2).

\section{CONClusions}

The design and practical implementation of NCSs are usually subject to the needs of both the control and communication performances. In order to achieve the optimized balance between the QoC of the NCS and the QoP of the communication network, we design the PDV-based packet length allocation scheme for NCSs. By comparing the scheme with conventional control algorithms and the packetbased control approach, we successfully demonstrate the effectiveness of the proposed scheme. Furthermore, we show that conventional control algorithms and the packet-based control approach can be regarded as two extreme cases of the PDV-PLA scheme, thus making the latter a unified framework for NCSs in terms of the usages of the communication resources.

\section{REFERENCES}

[1] R. Borges, R. Oliveira, C. Abdallah, and P. Peres, "Robust $H_{\infty}$ networked control for systems with uncertain sampling rates," IET Control Theory Appl., vol. 4, no. 1, pp. 50-60, 2010.

[2] M. Cloosterman, L. Hetel, N. van de Wouw, W. Heemels, J. Daafouz, and H. Nijmeijer, "Controller synthesis for networked control systems," Automatica, vol. 46, no. 10, pp. 1584-1594, 2010.

[3] A. Ulusoy, O. Gurbuz, and A. Onat, "Wireless model-based predictive networked control system over cooperative wireless network," IEEE Trans. Ind. Inform., vol. 7, no. 1, pp. 41-51, 2011.

[4] H. Gao, X. Meng, and T. Chen, "Stabilization of networked control systems with a new delay characterization," IEEE Trans. Autom. Control, vol. 53, no. 9, pp. 2142-2148, 2008.

[5] J. Sun, G.-P. Liu, J. Chen, and D. Rees, "Improved delay-rangedependent stability criteria for linear systems with time-varying delays," Automatica, vol. 46, no. 2, pp. 466-470, 2010.

[6] D. Xie, X. Chen, L. Lü, and N. Xu, "Asymptotical stabilisability of networked control systems: Time-delay switched system approach," IET Control Theory Appl., vol. 2, no. 9, pp. 743-751, 2008.

[7] R. Wang, G.-P. Liu, W. Wang, D. Rees, and Y.-B. Zhao, " $H_{\infty}$ control for networked predictive control systems based on switched Lyapunov function method," IEEE Trans. Ind. Electron., vol. 57, no. 10, pp. 3565-3571, 2010.

[8] S.-L. Dai, H. Lin, and S. S. Ge, "Scheduling-and-control codesign for a collection of networked control systems with uncertain delays," IEEE Trans. Control Syst. Technol., vol. 18, no. 1, pp. 66-78, 2010.

[9] E. Fridman, "A refined input delay approach to sampled-data control," Automatica, vol. 46, no. 2, pp. 421 - 427, 2010.

[10] Y.-B. Zhao, G.-P. Liu, and D. Rees, "Integrated predictive control and scheduling co-design for networked control systems," IET Control Theory Appl., vol. 2, no. 1, pp. 7-15, 2008.

[11] — " "Design of a packet-based control framework for networked control systems," IEEE Trans. Control Syst. Technol., vol. 17, no. 4, pp. 859-865, 2009.

[12] - "Packet-based deadband control for Internet-based networked control systems," IEEE Trans. Control Syst. Technol., vol. 18, no. 5, pp. 1057-1067, 2010.

[13] ITU-T, ITU-T Recommendation Y.1540, The ITU Telecommunication Standardization Sector (ITU-T) Std., 2002. [Online]. Available: http://www.itu.int/

[14] Y.-B. Zhao, G.-P. Liu, and D. Rees, "Actively compensating for data packet disorder in networked control systems," IEEE Trans. Circuits Syst. II-Express Briefs, vol. 57, no. 11, pp. 913-917, 2010. 